\title{
Laser desorption jet-cooling spectroscopy of para-amino benzoic acid monomer, dimer, and clusters
}

\author{
Gerard Meijer, Mattanjah S. de Vries, Heinrich E. Hunziker, and H. Russell Wendt \\ IBM Research Division, Almaden Research Center, San Jose, California 95120-6099
}

(Received 26 December 1989; accepted 2 March 1990)

\begin{abstract}
The technique of laser desorption followed by jet cooling allows wavelength-selective as well as mass-selective detection of molecules, desorbed from a surface without fragmentation.

Resonance enhanced multi photon ionization (REMPI) spectra of the para-amino benzoic acid (PABA) molecule and its methyl and $n$-butyl ester were obtained in this way. The origin of the $S_{1} \leftarrow S_{0}$ transition in PABA was found at $34173 \pm 2 \mathrm{~cm}^{-1}$. The adiabatic ionization potential of PABA was determined as $7.998 \pm 0.001 \mathrm{eV}$. In addition, jet-cooled REMPI spectra of the PABA dimer and its ring-deuterated isotopes were recorded. The dimer is formed by two identical hydrogen bonds between the carboxylic acid groups. The excitation in the dimer is found to be almost completely localized in one monomer unit. Clusters of PABA molecules with molecules seeded in the beam (argon, methanol, water) were resonantly detected as well, using PABA as the chromophore.
\end{abstract}

\section{INTRODUCTION}

In the last couple of years several groups have been studying laser desorption of organic molecules from surfaces. ${ }^{1-4}$ It is generally believed that under conditions of relatively low desorption laser power more neutrals than ions are produced in the desorption process. ${ }^{5-7}$ In order to study these neutrals, it is necessary to separate the detection step from the desorption step. In the most widely used approach the desorbed molecules are ionized shortly after they leave the surface, either by electron impact or by photoionization. The molecular ions are extracted and mass-selective detection is performed. As an analytical tool, this technique has found applications in several fields. It is used to determine the composition of bulk samples and surface layers. Compared with other surface analytical methods, the sensitivity of this technique is very high and small fractions of a monolayer can be detected. The selectivity of this process resides exclusively in the mass-selective detection. When electron impact is used for ionization, additional information is obtained from the often well-known fragmentation patterns. When photoionization is used, the wavelength of the ionization laser can be chosen such as to preferentially ionize a certain class of compounds. ${ }^{8,9}$

Although the mass resolution can be made high enough to determine the masses of biomolecules (masses up to $10000 \mathrm{amu}),{ }^{10,11}$ one would often like to have an additional dimension of selectivity, to pick a specific molecule from a complex mixture. Selective two-step ionization, using an electronic resonance in the molecules to be detected, gives such an additional selectivity. It is well known that even for large organic molecules with low symmetries, sharply resolved, fingerprint ionization spectra can be obtained when the rotational and vibrational degrees of freedom are cooled down. ${ }^{12}$ For molecules with a sufficiently high vapor pressure, this can be achieved by seeding them in the drive gas of a supersonic jet expansion, whereby cooling to a few degrees $\mathrm{K}$ can be obtained. In order to apply jet cooling to molecules with a lower vapor pressure, a different technique for mixing the molecules with the drive gas is required. In some cases heating of the nozzle can be used to increase the vapor pressure; however, pyrolysis often occurs. Laser desorption as a means to volatilize surface adsorbants has been demonstrated to circumvent pyrolysis as well as surface reactions. ${ }^{6,7}$

Recently it has been shown that laser desorption can be combined with jet cooling to obtain both mass-spectra and wavelength-spectra of complex molecules with a low vapor pressure. ${ }^{13-15}$ In our setup, laser desorption is performed very close to the orifice of a supersonic jet expansion. In this approach, the internal degrees of freedom of the desorbed molecules are cooled by multiple collisions in the expansion region. The desorbed molecules are entrained in the jet, they are carried downstream by the drive gas in a compact cloud, and are therefore brought into the detection region with a high efficiency. ${ }^{16}$ The jet cooling enables an efficient and selective ionization of the desorbed molecules when the wavelength of the ionization laser is chosen to be resonant with a vibronic transition. This makes it possible to detect a specific molecule amidst a soup of other, slightly different, molecules. In addition to its analytical usefulness, this technique is the only one that permits studying the gas-phase spectroscopy of a large number of involatile organic molecules.

In this article we demonstrate the power of laser desorption jet-cooling spectroscopy. In our setup rotational cooling down to $5-10 \mathrm{~K}$ is obtained, ${ }^{17}$ with a detection limit of less than $10^{6}$ molecules in the desorption laser-spot. ${ }^{18}$ Laser desorption jet-cooling spectroscopy is applied to the para-amino benzoic acid (PABA) molecule and clusters associated with it. The PABA molecule is of particular interest because it is closely related to both aniline and benzoic acid. Both these molecules are sufficiently volatile to be seeded in a beam, and their gas-phase spectroscopy is well known. ${ }^{19-21}$ PABA, however, has a vapor pressure of $10^{-7}$ Torr at room temperature, ${ }^{22}$ and nothing has been known about its gasphase spectroscopy until now, despite its widespread use as a 
sunscreen and as a photodegradation inhibitor. The spectrum of the PABA dimer reflects unambiguously the geometrical structure of the dimer, and this will be discussed in detail below. In addition to detecting clusters of desorbed molecules with themselves, clusters of desorbed molecules with molecules seeded in the molecular beam were formed, cooled, and resonantly detected.

\section{EXPERIMENTAL}

The experimental setup is schematically shown in Fig. 1. The sample is mounted just in front of a pulsed valve (General Valve) with a $0.5 \mathrm{~mm}$ nozzle diameter. The sample surface is brought very close to the lower edge of the nozzle (within $0.1 \mathrm{~mm}$ ). The height of the sample can be varied continuously. Typically the sample is $0.5-2.0 \mathrm{~mm}$ below the molecular beam axis. The desorption laser hits the sample from above, and the position of the desorption laser spot relative to the nozzle can be adjusted. A telescope providing a view along the desorption laser path aids in the alignment. Obviously, the desorption laser spot has to be centered on the molecular beam axis, whereas the distance from the nozzle can be chosen anywhere between $0.5-5$ nozzle diameters. The sample is mounted on a rail, so that a fresh part of the sample can be brought in front of the nozzle whenever it is needed. For the experiments to be discussed here, a $3 \times 5 \times 40 \mathrm{~mm}$ piece of fritted glass was used as sample holder. PABA as well as its alkyl derivatives were commercially obtained, and used without further purification. The PABA was dissolved in methanol, and the solution was brought on the fritted glass. After evaporation of the methanol a thin layer of PABA covered the fritted glass. In all experiments, Ar, with a backing pressure of 5-8 atm, was used as the drive gas.

In some experiments partly deuterated PABA was used. We synthesized PABA with $D$ atoms in the ortho position to the $\mathrm{NH}_{2}$ group by heating under reflux for fifty hours a slightly acidic $(p H=4)$ solution of PABA in $\mathrm{D}_{2} \mathrm{O}$. This also substituted the outer $\mathrm{H}$ atoms for $\mathrm{D}$ atoms but these latter ones were easily resubstituted by dissolving the deuterated compound in methanol, and subsequent recristallization. In this way we obtained doubly ring-deuterated PABA (mass 139 ) with a $95 \%$ purity.

For desorption a laser is required that heats the substrate at a high enough rate to cause thermal desorption, without causing photolysis and/or pyrolysis. We used a $\mathrm{KrF}$ ( $248 \mathrm{~nm}$ ) excimer laser (Lumonics Hyperex-400; unstable resonator optics) for the desorption process. A $2 \mathrm{~mm}$ diameter spot was spatially cut out of the excimer beam profile, a few meters away from the laser. The intensity of the resulting beam was reduced by inserting calibrated attenuation filters to typically $0.1 \mathrm{~mJ}$, in a $15 \mathrm{~ns}$ pulse. This light was loosely focused onto a $0.3 \mathrm{~mm}$ diameter desorption spot on the sample. Although we know that some photochemistry was going on at or near the surface (we saw for instance aniline being produced by irradiating PABA with $248 \mathrm{~nm}$ ) this was not to an extent that it influenced our data. Identical spectra and a similar monomer/dimer ratio in the beam were observed when a XeF ( $351 \mathrm{~nm})$ excimer laser was used for the desorption process. Timing of the desorption laser pulse in the 150

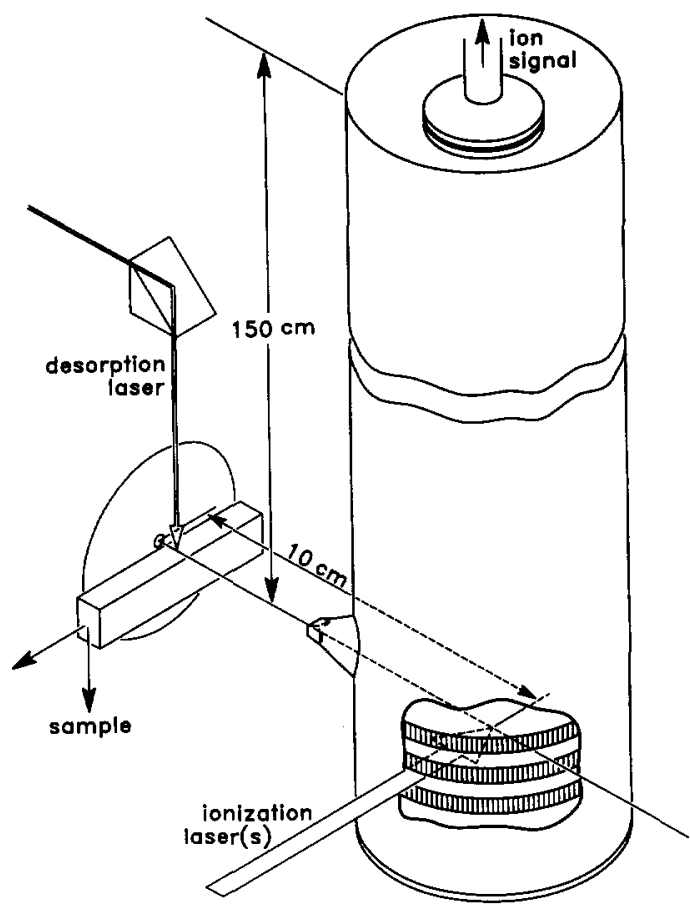

FIG. 1. Schematic view of the experimental setup. See the text for details.

$\mu \mathrm{s}$ long gas pulse as well as the timing of the ionization laser(s) was controlled by a pair of four-channel digital delay generators (Stanford Research Systems, Inc. model DG 535). The experiments ran at $10 \mathrm{~Hz}$.

Following desorption, the desorbed molecules are entrained in the jet and a fraction ${ }^{16}$ passes through a $1 \times 4 \mathrm{~mm}$ slit skimmer, with the slit in the horizontal direction, $2.3 \mathrm{~cm}$ away from the nozzle. Thus the beam enters the differentially pumped ionization chamber. The walls of the ionization chamber can be cooled to liquid nitrogen temperature to reduce the ion signal that is due to ionization of background gases. Ionization takes place at a $10 \mathrm{~cm}$ distance from the nozzle, between the extraction grids of a Wiley-McLarentype $^{23}$ linear time-of-flight mass spectrometer (R. M. Jordan Co.). As nonselective ionization source another excimer laser, operating on $\mathrm{KrF}$ ( $248 \mathrm{~nm}$ ), $\mathrm{ArF}$ ( $193 \mathrm{~nm}$ ) or $\mathrm{F}_{2}$ (157 $\mathrm{nm}$ ) can be used. Nonresonant ionization can be very useful for optimizing the desorption process. For resonant ionization the frequency-doubled output of a Quanta-Ray and/or Lumonics dye laser, pumped by the second and third harmonic, respectively, of a Nd:YAG laser, is used.

The positive molecular ions are created in a $200-250$ $\mathrm{V} / \mathrm{cm}$ electrical field, and pass through a $150 \mathrm{~cm}$ long, grounded drift tube before they are collected on a 2" dual channel plate. The overall ion transmission of this TOF mass spectrometer is measured to be about $40 \%$. The main ion loss is probably due to fringe fields penetrating between the extraction grids and into the field-free region of the drift tube, thus causing ions to be deflected outwards. The dual channel plates amplify the ion signal by a factor of $10^{7}$. A home-built, fast preamplifier is connected as close as possi- 
ble to the channelplates and amplifies the signal by another factor of 10 , before it is displayed on a digital oscilloscope (LeCroy 9400). The mass spectra displayed and stored in the scope are read out by an IBM PC. By adjusting the voltages on the extraction grids to fulfill the Wiley-McLaren conditions, the ion peaks can be made as narrow as $30 \mathrm{~ns}$ (FWHM), on a total flight-time of several tens of $\mu \mathrm{s}$. This corresponds to a mass resolution $\Delta M / M$ of $1 / 300$ at the PABA mass (137 amu).

The procedure for recording wavelength spectra is as follows: A mass spectrum is read out by the PC, and integration windows are set over several (maximum of five) mass intervals. The wavelength scan of the ionization laser is controlled by the PC. At every wavelength setting a set of typically 10 mass spectra are recorded and averaged. Then the 10 $\mathrm{Hz}$ sequence is interrupted, the ionization laser steps to its next wavelength, and the averaged ion signal in the various mass windows is calculated and displayed. The scan goes on over a preset wavelength interval, and at the end, wavelength spectra for the different masses are displayed. This final set of spectra is stored into memory.

\section{RESULTS AND DISCUSSION}

\section{A. REMPI Of PABA}

As mentioned before, nothing has been known about the gas-phase spectroscopy of PABA until now. To get an idea where to look for the lowest absorption band, solution spectra can be used. In order to locate the origin of the electronic transition in the gas-phase more precisely, we recorded a room-temperature $(1+1)$-REMPI spectrum. Unless indicated otherwise, all spectra were obtained using the same laser for resonant excitation and ionization (one color, two photon ionization). With the walls of the ionization chamber at room temperature, there was approximately $10^{-8}$ Torr vapor pressure of PABA in the machine, enough to detect its REMPI spectrum. The result is shown in Fig. 2. The spectrum is recorded by monitoring ions at the parent mass (137 amu), and is not corrected for the wavelength dependence of the ionization laser power. The structure is due to resonance absorption into the first electronically excited singlet state which is analogous to the ${ }^{1} B_{2 u}$ state in benzene. We assume that ionization out of this state does not add any structure in this wavelength region. It is interesting to compare the broad bands that show up in this spectrum with the much sharper structures in the room-temperature laser-excited fluorescence spectrum of aniline ${ }^{19,20}$; there is much more spectral congestion for the PABA molecule.

The $(1+1)$-REMPI spectrum of the laser-desorbed, jet-cooled PABA is shown in Fig. 3. The origin of the electronic transition is located at $292.63 \mathrm{~nm}$ (34 173 \pm 2 $\mathrm{cm}^{-1}$ ). A large number of transitions to vibrational levels in the excited electronic state appear in the spectrum. To the red side of the $0-0$ band hardly any structure is observed, and, from the absence of hot bands, we estimate the vibrational temperature to be less than $30 \mathrm{~K}$. A more detailed study of the cooling characteristics of this machine has been performed on anthracene, a molecule whose spectroscopy is well known. These measurements indicate a rotational tem-

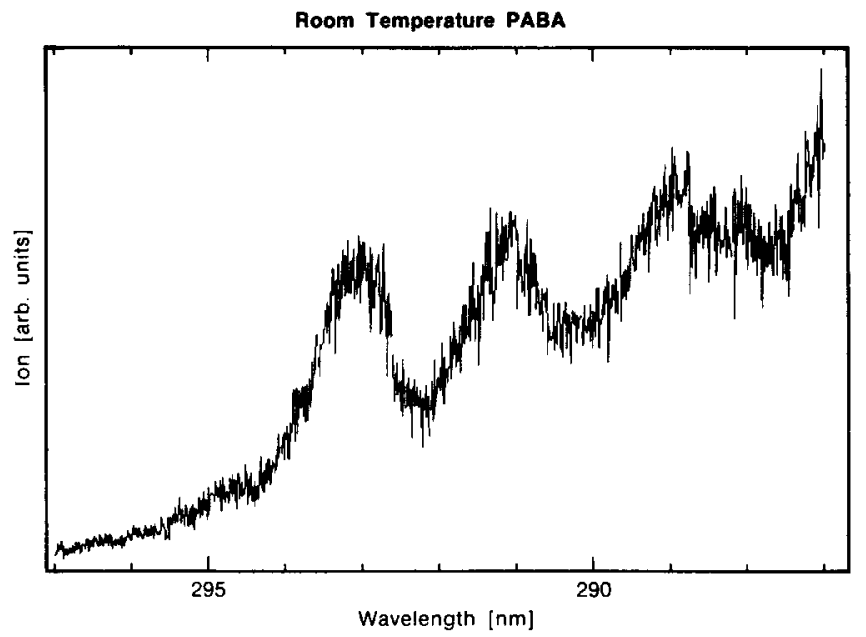

FIG. 2. $(1+1)$-resonance enhanced multiphoton ionization spectrum of para-amino benzoic acid at room temperature. Ions are detected at the parent mass ( $137 \mathrm{amu}$ ).

perature between 5 and $10 \mathrm{~K} .{ }^{17}$ The energy of each major peak in the spectrum of Fig. 3 with respect to the electronic origin is indicated, in $\mathrm{cm}^{-1}$, above each peak. Contrary to the observations in the methylbenzoate and the benzoic acid spectra, ${ }^{21}$ no sign of a rotational isomer of PABA is seen. Although a complete assignment of the vibrational spectrum of PABA does not yet exist, the first strong vibration at $\mathbf{4 6 1 . 2}$ $\mathrm{cm}^{-1}$ can be ascribed to the lowest-energy active ring vibration, corresponding to the 6 a vibration in benzene. As will be seen from a comparison of the spectrum in Fig. 3 with the spectra of several alkyl esters of PABA, the next strong vibrations at $550.4,812$, and $995 \mathrm{~cm}^{-1}$ are ring vibrations as

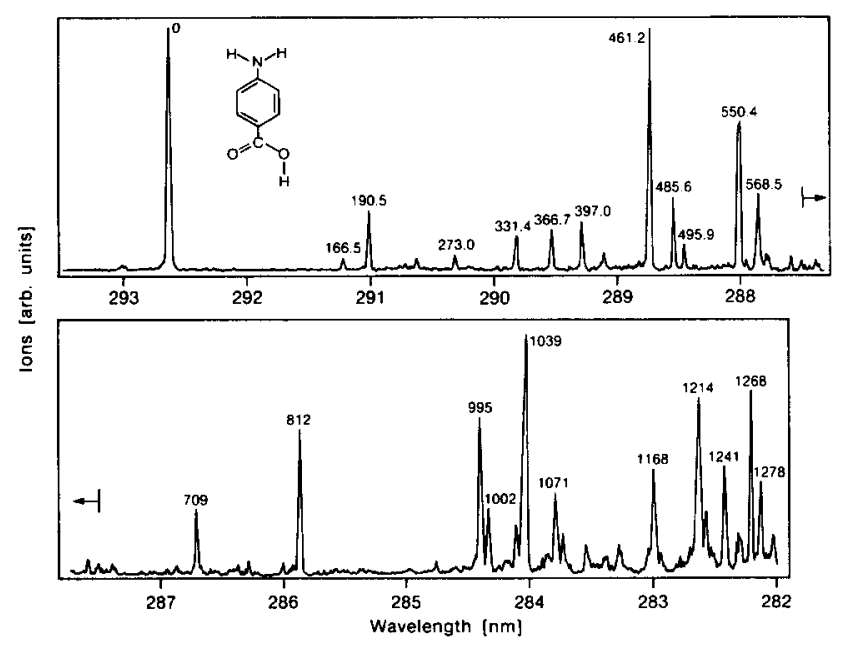

FIG. 3. $(1+1)$-REMPI spectrum of laser desorbed jet-cooled PABA. The width of the $0-0$ transition is about $3 \mathrm{~cm}^{-1}$ (FWHM), due to unresolved rotational structure. Frequency distances of each peak from the origin are indicated in $\mathrm{cm}^{-1}$. The accuracy is $\pm 1 \mathrm{~cm}^{-1}$ in the upper panel and \pm 2 $\mathrm{cm}^{-1}$ in the lower panel. 
well. The lower energy vibrations involve motions of the substituent groups with respect to the ring. Since such low energy vibrations are absent in the aniline spectrum, they are most probably due to the $\mathrm{COOH}$ substituent.

Considerable caution is necessary in comparing the intensities of the various peaks that show up in the spectrum. First of all, there is the wavelength dependence of the ionization laser power, which in principle can be corrected for. A major problem, however, is the pulse-to-pulse fluctuation in the amount of desorbed material injected into the jet. This is partly due to depletion of the sample spot in time, but mainly to the fact that small fluctuations in desorption laser power give rise to large fluctuations in the amount of material desorbed. ${ }^{16}$ When the wavelength of the ionization laser was set on the 0-0 transition of PABA for instance, typical shotto-shot fluctuations of $30 \%$ occurred in the ion signal. The spectrum in Fig. 3 was taken by averaging over 10 shots. A new spot was used after every 2000-3000 shots and we believe that the relative intensities are at least qualitatively right.

The spectrum in Fig. 3 was recorded by measuring only the parent ions, at mass 137 amu. As shown in Fig. 4, there was some fragmentation, even at relatively low ionization energy. The mass spectrum in Fig. 4 was recorded with a ionization laser fluence of $10 \mathrm{~mJ} / \mathrm{cm}^{2}$, at the wavelength of the PABA 0-0 transition. The major fragmentation peaks are at $120 \mathrm{amu}$ and at $92 \mathrm{amu}$, corresponding to the loss of an $\mathrm{OH}$ group and of an $\mathrm{COOH}$ group, respectively. The mass resolution was high enough to separate the PABA containing the C-13 isotope at mass 138 from the main peak at 137 amu. It should be noted that the isotope ratio inferred from the REMPI mass spectra does not necessarily reflect the actual abundance of the isotopes, because of the isotope shift in their wavelength spectra. We could also clearly separate mass 92 from mass 93 . There was no way, however, in which we could resolve the apparent double peak at mass 120-121. This indicates that the loss of the $\mathrm{OH}$ group takes place in the molecular ion, and is relatively slow; it is on the way out of the extraction region that the PABA ion looses its hydroxyl part. For the methyl ester of PABA (methyl 4-amino benzoate) we saw a similar fragmentation pattern, i.e., loss of the $\mathrm{OCH}_{3}$ group and loss of the $\mathrm{COOCH}_{3}$ group. In this case, however, the fragmentation peak at mass 120 was a sharp, single peak, which indicates a faster fragmentation process. [One might have expected the energy to flow slower into the dissociating bond for the methyl ester of PABA, as this larger molecule has more internal degrees of freedom than the PABA molecule. There are several other effects, however (i.e., change in excess energy in going from PABA to substituted PABA, change of the bond strength in going from the acid to the ester) that might explain the observed, faster, dissociation process.] The fragmentation pattern was identical for all the wavelength peaks in the spectrum of Fig. 3. In general, the 0-0 transition showed a little bit less fragmentation that the other transitions. Of course, when considerably higher ionization laser power was used, fragmentation was much more severe, and a lot of smaller fragments appeared.

The spectra we discussed thus far have all been taken using one laser for both the resonant excitation and the ioni-

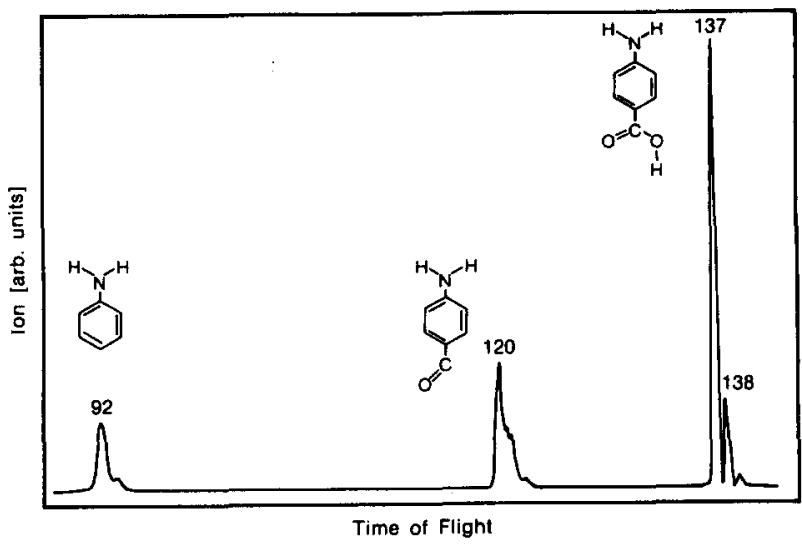

FIG. 4. TOF spectrum, showing the fragmentation pattern of PABA. The ionization laser is set at the 0-0 transition. Laser fluence (unfocused beam) is $\sim 10 \mathrm{~mJ} / \mathrm{cm}^{2}$.

zation. To determine the ionization potential (IP) of PABA we used two separate dye lasers. The dye laser used to perform resonant excitation can be reduced in intensity. The dye laser used for ionization can be quite intense, as three photons of this laser are needed for nonresonant ionization, whereas only one photon is needed to ionize from the intermediate state. As mentioned earlier, a general problem with the laser desorption jet cooling technique is the fluctuation of the signal. This makes two-color experiments hard to perform, because they require a measurement in the presence of a large and fluctuating background. An additional problem in the case of the PABA molecule is the short lifetime of the intermediate singlet state. It turned out that a mere overlapping of the two dye lasers in time (to a ns precision) was not enough to produce a strong two-color ionization signal. Even with the resonant excitation laser an order of magnitude weaker than the ionization laser, the $(1+1)$-REMPI signal and the two-color ionization signal were comparable in intensity. This is due to the jagged temporal shape of the pump pulse from our (non-injection-seeded) Nd:YAG laser, which causes intensity spikes in the output of the dye lasers. As both dye lasers are pumped by the same Nd:YAG laser, the overlap of the spikes can be changed by varying the path length of one laser with respect to the other. By changing this path length over a few $\mathrm{cm}$, a major change in the total ion yield was observed. From this we estimate that the lifetime of the intermediate state in the PABA molecule is on the order of a few hundred ps. Once the optimum overlap between the excitation laser and the ionization laser was found, the onset of ionization could be determined. In the inset of Fig. 5 a typical ionization onset is shown. The resonant excitation laser was fixed in wavelength and excited the $0-0$ of PABA at $34173 \mathrm{~cm}^{-1}$. Ions of mass 137 were collected as the ionization laser was scanned around $330 \mathrm{~nm}$. The total ion signal at mass 137 increased by an order of magnitude once the wavelength of the ionization laser was short enough to cause ionization out of the intermediate state. Several spectra showing the onset of ionization were recorded as 


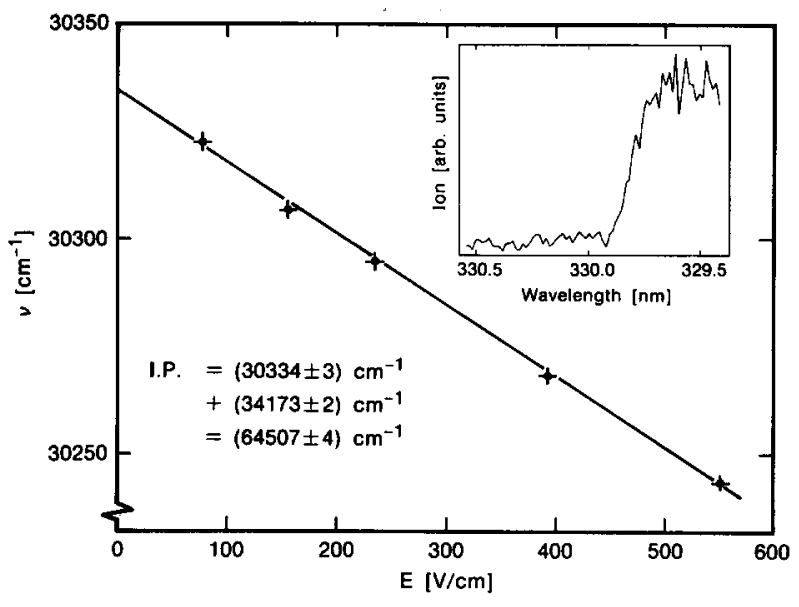

FIG. 5. Energy needed to ionize PABA out of the $S_{1}$ state as a function of the electric field in which the ions are produced. In the inset a typical scan showing the onset of ionization is shown in an electric field of $155 \mathrm{~V} / \mathrm{cm}$. Extrapolating the curve to zero electric field, allows the ionization potential to be determined as $64507 \pm 8 \mathrm{~cm}^{-1}$.

a function of the electric field in which the ions are produced. When the wavelength of the ionization laser is too high to cause photoionization, it can still excite to one of the numerous Rydberg states. Electric field ionization of these highlying Rydberg states causes an apparent lowering of the ionization potential. ${ }^{24}$ Figure 5 shows the electric field dependence of the ionization onset. A lowering of the ionization potential by about $18 \pm 1 \mathrm{~cm}^{-1}$ per $100 \mathrm{~V} / \mathrm{cm}$ of electric field is seen. The shape of the curve we obtained is similar to the electric field dependence found for benzene and anthracene. ${ }^{24}$ Extrapolation of the curve in Fig. 5 to zero electric field allows the ionization potential to be determined as $64507 \pm 8 \mathrm{~cm}^{-1}$, where the error is equal to two times the standard deviation.

A schematic energy level diagram of PABA is shown in Fig. 6. The first excited singlet state lies higher than halfway to the IP, which makes single-color $(1+1)$-REMPI detection possible. Note that the energy of the $F_{2}$ laser $(157 \mathrm{~nm})$ is just not enough to cause one-photon ionization of the PABA monomer. We already noted that the $S_{1}$ state has a short lifetime. A substantial fraction of the population in the $S_{1}$ state undergoes intersystem crossing to a long-lived state, probably the lowest lying triplet state, the $T_{1}$ state. We could probe the population in this triplet state by delayed ionization with the ArF (193 nm) laser. The delay time could be as long as several $\mu$ s without a significant reduction in signal; the main reason for the observed decrease in ion signal for longer delay times was due to triplet state PABA molecules leaving the ionization region. Although the energetic position of the triplet state is not known, we can give some upper and lower limits. The triplet state can be detected by the ArF laser $(6.42 \mathrm{eV})$ but not by the $\mathrm{KrF}$ laser $(5.00 \mathrm{eV})$. If we assume there is no change of vibrational energy in the ionization process, this places the triplet state between 1.57 and $3.00 \mathrm{eV}$ above the singlet ground state. Some spectra were recorded in which the population in the triplet state was

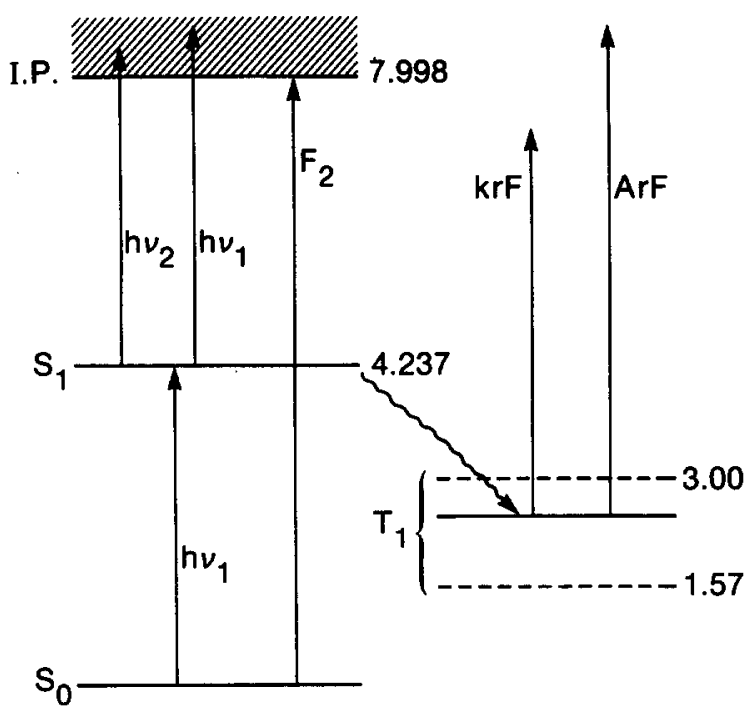

FIG. 6. Schematic energy level diagram of the PABA monomer. Energy scale is in $\mathrm{eV}$.

detected while the excitation laser was scanned over the $S_{1} \leftarrow S_{0}$ region. Due to the long lifetime of the triplet state simultaneous recording of the $(1+1)$-REMPI spectrum via the singlet and triplet states is possible: If the $\mathrm{ArF}$ laser is delayed by $300 \mathrm{~ns}$, the ions created out of the triplet state appear 300 ns later (appear at "another mass") than the ions created by the excitation laser directly out of the $S_{1}$ state (note that the resonant excitation laser cannot ionize out of the triplet). We measured both spectra simultaneously to see if there is a strong vibrational state dependence of the intersystem crossing rate. Simultaneous recording is important because it eliminates the main source of noise, i.e., any fluctuation in the amount of PABA present in the beam. The result was negative; for all the vibrations below $600 \mathrm{~cm}^{-1}$ (the rest of the spectrum was not investigated) the spectrum obtained by delayed ionization out of the triplet state was identical to the $(1+1)$-REMPI spectrum shown in Fig. 3 . The intensities are the same within $20 \%$, which means the intersystem crossing rate is constant to within $10 \%$.

The most efficient detection of PABA was found to be the direct one-color $(1+1)$-REMPI detection. Nevertheless we estimate the overall ionization efficiency to be only of the order of $1 \%$, mainly due to the short intermediate state lifetime. Experiments are under way to determine the lifetime of the intermediate state and the absolute ionization efficiency, to be able to quantify the absolute detection efficiency of PABA on a surface in our setup.

In Fig. 7 part of the $(1+1)$-REMPI spectrum of PABA (upper panel) is shown together with the corresponding spectra of methyl 4-amino benzoate (middle panel) and $n$-butyl 4-amino benzoate (lower panel). The spectra of the alkyl esters of PABA are only slightly redshifted with respect to the PABA spectrum. This slight shift is indicative of the fact that the excitation is mainly localized on the 


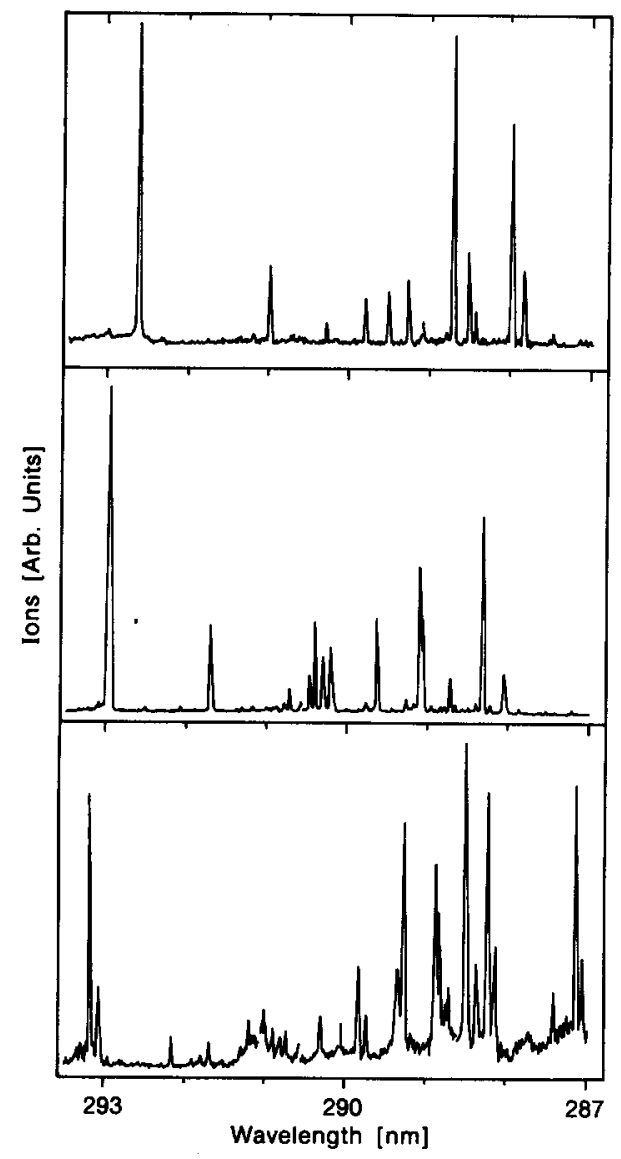

FIG. 7. Part of the $(1+1)$-REMPI spectra of laser desorbed jet-cooled PABA (upper), methyl 4-amino benzoate (middle) and $n$-butyl 4-amino benzoate (lower). In all spectra ions are collected at the parent mass. The $n$-butyl 4-amino benzoate spectrum shows the presence of two distinct types of rotational isomers, in an approximate 3:1 ratio.

other side of the molecule, i.e., on the amino group. When methyl groups are attached to the nitrogen, the spectrum changes completely. Sharp transitions appear only in a narrow energy range near the $0-0$ band, about $10 \mathrm{~nm}$ redshifted from the PABA origin. The vibronic structure indicates a different type of electronic transition. ${ }^{25-27}$

The major vibrations in the PABA spectrum are seen at the same frequencies in the spectra of the methyl ester and the $n$-butyl ester, indicating that these are ring vibrations, hardly influenced by the substituent groups. At lower frequencies a set of vibrations attributed to vibrations in the alkyl groups and to vibrations of the alkyl groups with respect to the frame appear in the spectrum. For the $n$-butyl 4amino benzoate an extra peak appears very close to the origin. A similar splitting is seen for most other peaks in this spectrum. This is indicative of the presence of two distinct types of isomers, as has been seen for many other molecules as well. ${ }^{28,29}$ The spectra shown in Fig. 7 demonstrate that laser desorption jet cooling spectroscopy works for these more complicated molecules as well.

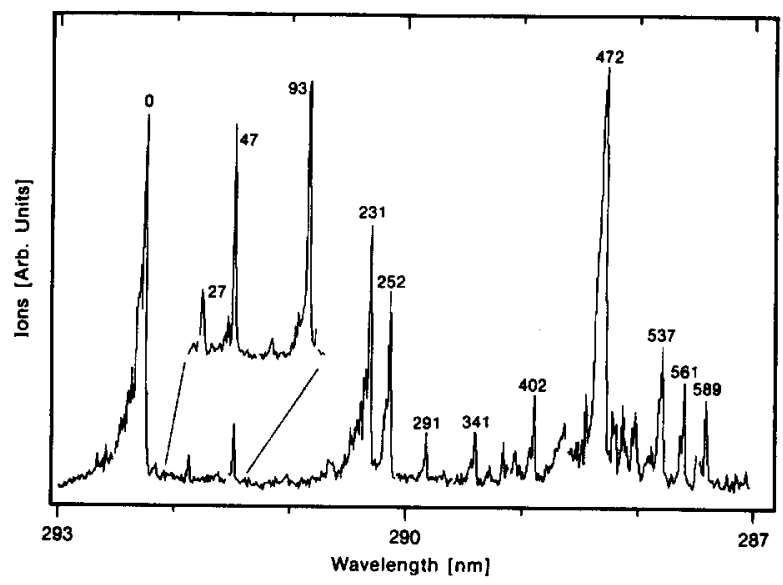

FIG. 8. $(1+1)$-REMPI spectrum of laser desorbed jet-cooled PABAdimers, detected at mass 274 . The electronic origin of the dimer spectrum is $42.0 \pm 0.8 \mathrm{~cm}^{-1}$ blueshifted with respect to the origin of the PABA-monomer. The linewidth of the electronic origin of the PABA-dimer is larger than for the monomer, and is about $7 \mathrm{~cm}^{-1}$. This might be due to a somewhat higher temperature for the dimer than for the monomer. Frequency distances from the various peaks in the spectrum to the electronic origin are indicated (in $\mathrm{cm}^{-1}$ ) above each peak, and are accurate within $\pm 1 \mathrm{~cm}^{-1}$. The low-lying hydrogen bond vibrations are shown on an expanded scale in the inset.

\section{B. Spectroscopic study of PABA dimers}

Under slightly different desorption conditions, intense spectra could be recorded at mass 274 , the mass of the PABA dimer. The changes necessary to get cooled PABA dimers in the beam can be qualitatively understood. To see any cluster formation in the gas phase, a high density of desorbed material is needed. This can in principle be obtained in two ways. One can increase the desorption laser fluence, but it turns out that jet-cooling deteriorates then. This is possibly due to an increase in translational energy of the desorbed molecules. The better way is to use a very low desorption laser fluence, just above threshold, and to move the sample closer to the molecular beam axis. In addition, the desorption spot has to be as close to the nozzle as possible, to get optimum cooling. To minimize problems with disturbance of the jet due to the presence of the sample, a high backing gas pressure turned out to be advantageous. We used $8 \mathrm{~atm}$ of Ar for this reason.

A typical dimer spectrum is shown in Fig. 8. This spectrum was recorded at mass 274 , the mass of the unfragmented dimer. When desorption conditions were optimized for the cooled dimer signal, its intensity was about $20 \%$ of the monomer signal. Upon excitation, the dimer partly dissociates into monomer fragments, thus its resonances can be seen at the monomer mass as well. Fragmentation increases with increasing detection laser fluence. The dimer $0-0$ band is blueshifted by $42.0 \pm 0.8 \mathrm{~cm}^{-1}$ with respect to that of the monomer. The dimer spectrum shows a structure more or less similar to that of the monomer spectrum, and ring vibrations of the monomer all show up with a similar blue shift in the dimer spectrum. There are, however, distinct differences 


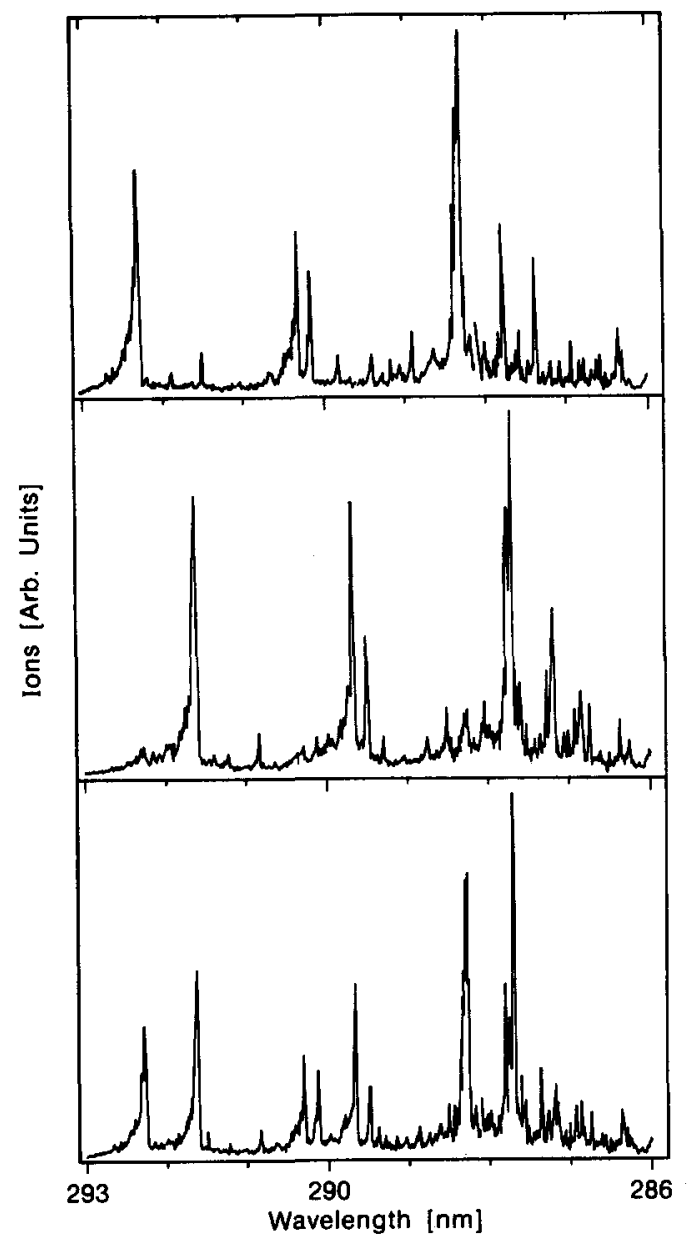

FIG. 9. $(1+1)$-REMPI spectra of PABA dimers (mass 274; upper) deuterated PABA dimers (mass 278; middle) and of mixed deuterated-undeuterated PABA complexes (mass 276; lower). All three spectra are recorded in the same laser scan. The lower spectrum shows that the excitation in the dimer is almost completely localized in the monomer units.

between the monomer and the dimer spectrum. First of all there are some low-lying vibrations in the dimer, which we ascribe to bending vibrations in the hydrogen bonds that keep the monomer parts together. These are shown enlarged in the inset of Fig. 8. Secondly, there is the anomalous behavior of the $190.5 \mathrm{~cm}^{-1}$ vibration of the monomer; this vibration seems to be absent in the dimer spectrum, which instead shows two strong peaks around 231 and $252 \mathrm{~cm}^{-1}$.

What is the geometrical structure of this dimer? For PABA this question is especially intriguing because it has an electron-donating and an electron-withdrawing side. It also has the possibility of forming an internal salt, which might be expected to be stable. Several geometries can be thought of, in which the $\mathrm{COOH}$ group is $\mathrm{H}$ bonded to the $\mathrm{NH}_{2}$ group. A "sandwich"-like structure (rings on top of each other in a symmetrical way, with the $\mathrm{NH}_{2}$ groups connected to the $\mathrm{COOH}$ groups) using two or even four hydrogen bonds can be constructed. On the other hand, a dimer structure identical to the well-known benzoic acid dimer ${ }^{30,31}$ held together by two hydrogen bonds between the $\mathrm{COOH}$ groups is also possible. From $x$-ray cristallographic data ${ }^{32}$ it is known that PABA forms this latter type of dimer in the crystal.

To unravel the geometrical structure of the free dimer, spectra were recorded of PABA complexes consisting of different monomer units. For this, a mixed sample of PABA and PABA- $d_{2}$ (the two $\mathrm{H}$ atoms in the ring ortho to the $\mathrm{NH}_{2}$ group substituted by D atoms; mass 139 ) was used. The spectrum of the PABA monomer hardly shows a shift when any of the amino or hydroxyl $H$ atoms are substituted by $a D$ atom. Such a spectral shift is needed when one wants to label one monomer in the dimer. Substitution of one $\mathrm{H}$ atom in the ring by a $\mathrm{D}$ atom, however, gives a spectral blueshift of about $38 \mathrm{~cm}^{-1}$. This is the usual shift caused by the reduction of the C-H (C-D) stretch frequency in the excited state. (It is worth mentioning at this point that PABA containing the $\mathrm{C}$ 13 isotope, seen at mass 138 in Fig. 3, shows a spectral blue shift of a few wave numbers with respect to PABA, due to the same effect.)

In Fig. 9 spectra obtained using a 1:1 mixture of PABA (mass 137) and PABA- $d_{2}$ (mass 139) are shown. The spectra of the three different kind of dimers, i.e., $d_{0}-d_{0}, d_{2}-d_{0}$ and $d_{2}-d_{2}$, were recorded in the same laser scan. The top spectrum shows the $d_{0}-d_{0}$ dimer spectrum, detected at mass 274 . The middle spectrum is that of the $d_{2}-d_{2}$ dimer (mass 278) and all peaks show the same shift with respect to the undeuterated dimer as that of the deuterated monomer with respect to the undeuterated monomer, i.e., $76 \pm 2 \mathrm{~cm}^{-1}$. The lower spectrum was recorded at mass 276 of the mixed $d_{2}-d_{0}$ dimer, and it is almost exactly equal to the sum of the first two spectra. From this it can be concluded that the excitation is localized in one of the monomer units; either one half or the other is excited in the loosely coupled dimer.

Several laser scans were made to examine the region around the electronic origins of the three different types of PABA complexes in more detail. A higher resolution study revealed that there are minor shifts in the positions of the origins. The $d_{0}-d_{0}$ dimer $0-0$ band is $0.23 \pm 0.17 \mathrm{~cm}^{-1}$ blueshifted with respect to the corresponding origin in the mixed $d_{2}-d_{0}$ dimer. The $d_{2}-d_{2}$ dimer $0-0$ band is $0.59 \pm 0.17 \mathrm{~cm}^{-1}$ blue-shifted with respect to the corresponding origin in the mixed $d_{2}-d_{0}$ dimer. Following the approach outlined by Baum and McClure ${ }^{33-36}$ for the benzoic acid dimer, the observed spectral shifts can be explained when two different effects are taken into account. First, there is the zero-point shift $\Delta$ in the band origin, due to deuteration of the unexcited half of the dimer. Secondly, there is the electronic interaction energy $\epsilon$ between the two monomer units of the dimer. This causes an exciton splitting, but only a transition (onephoton) to one of the two sites is symmetry allowed in the homogeneous dimer. When second-order effects are neglected, the interaction energy causes an appreciable shift only for the dimer consisting of identical monomer units. In this approximation, the splitting between the origin in the $d_{0}-d_{0}$ dimer and the corresponding origin in the mixed $d_{2}-d_{0}$ dimer is equal to $\epsilon-\Delta$, whereas the origin in the $d_{2}-d_{2}$ dimer is higher by $\epsilon+\Delta$ than the corresponding origin in the mixed $d_{2}-d_{0}$ dimer. From this we find a small, positive value for the interaction energy, equal to $\epsilon=0.4 \pm 0.3 \mathrm{~cm}^{-1}$, 


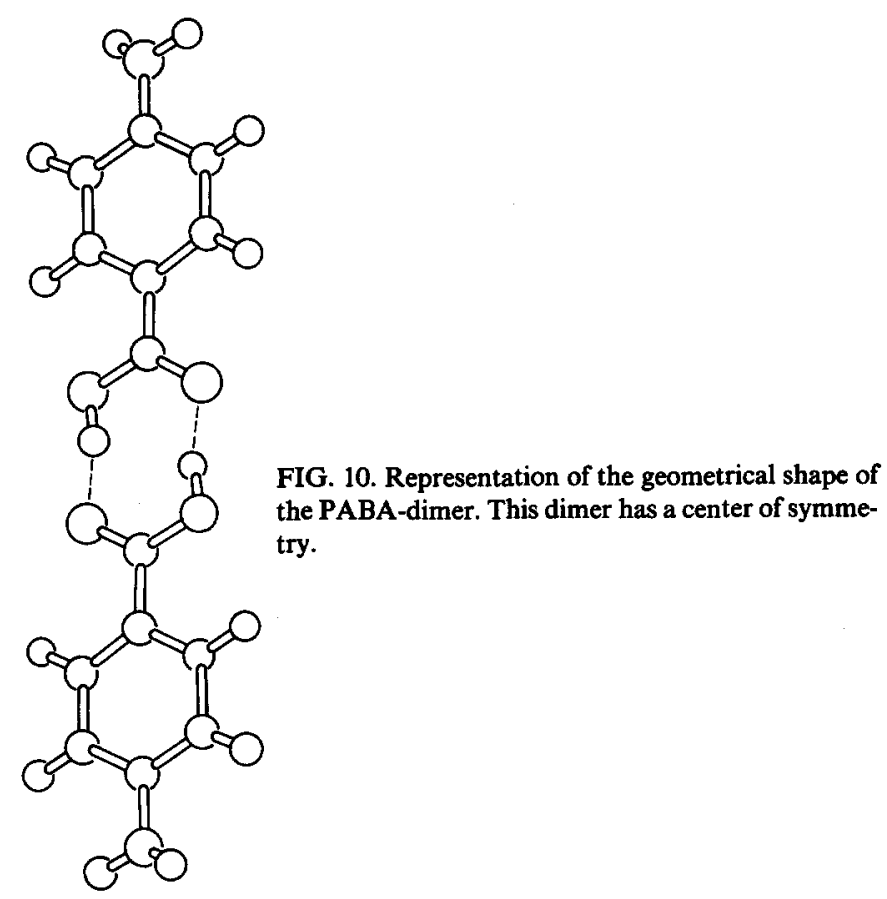

where the error is two times the standard deviation.

From the fact that the spectrum for the mixed deuterated-undeuterated PABA complex splits into two distinct parts, it immediately follows that the homogeneous PABA dimer is symmetrical. Only when both monomer parts in the dimer are completely identical the appearance of only one spectrum can be explained. If both parts were not identical, one of them would act as a proton donor and the other as a proton acceptor, and again we would see a double spectrum. This argument excludes a "head-to-tail" dimer, in which one $\mathrm{NH}_{2}$ group is H-bonded to a $\mathrm{COOH}$ group. It also excludes a dimer in which the $\mathrm{NH}_{2}$ groups are hydrogen-bonded together, as there is no symmetrical way of doing this. Still, there are several symmetrical structures possible. First, there is the symmetrical structure in which the rings are in a staggered, "sandwich"-type configuration, each having its $\mathrm{NH}_{2}$ group connected to the others' $\mathrm{COOH}$ group. Configurations of this type having two or even four hydrogen bonds can be thought of. In this case, however, one would expect a much larger interaction between the monomer units. This leaves us with the symmetrical dimer, as in benzoic acid, in which the $\mathrm{COOH}$ groups are connected by two hydrogen bonds. A strong indication for this structure being the correct one is the fact that the interaction energy in the benzoic acid dimer ${ }^{36}$ is similar to the one we find for the PABA dimer. Using the dipole-dipole coupling approximation a small positive value for the interaction energy is obtained when the rings are held together in this way. ${ }^{36}$ An argument against this structure might be that the benzoic acid dimer shows a red shift relative to the benzoic acid monomer, whereas we see a blue shift upon dimerization. However, the sign of the shift upon dimerization depends critically on the electron donating and accepting capabilities of the carboxyl group. These quantities are certainly changed substantially when an amino group is placed in the para-position.
We conclude that our experimental data favor a geometrical structure of the PABA-dimer in the gas phase as depicted in Fig. 10, similar to the structure in the crystal. ${ }^{32}$ As a check we recorded the spectrum of the complex of PABA with $4 \mathrm{~N}$ (di-methyl) benzoic acid. This complex showed up with an intensity that was comparable with that of the PABA-dimer, when a sample was used with a 1:1 ratio of PABA and 4 N(di-methyl) benzoic acid. It has a resonance at the same position as the PABA-dimer. Since in this case the amino side is blocked this leaves no doubt that the complex is formed by hydrogen bonding between the carboxyl groups.

Just as we did for the monomer, we also tried to determine the ionization potential for the dimer. Using two dye lasers, it was difficult to determine where ionization started. However, the ionization potential of the dimer was lower than that of the monomer by at least $0.1 \mathrm{eV}$. To confirm this, we used the $F_{2}(157 \mathrm{~nm})$ excimer laser for nonresonant, single photon ionization. As is indicated in Fig. 6, the $F_{2}$ laser cannot ionize the monomer with one photon. The dimer could be ionized with one photon, which puts the dimer IP below $7.90 \mathrm{eV}$. When the $\mathrm{F}_{2}$ laser was used to ionize the dimer, we could not expect to see fragmentation of the dimer into a charged monomer unit and a neutral one, as the $F_{2}$ laser energy is too low to ionize the PABA monomer. We did see some dimer fragments at mass 138 instead, due to formation of a charged $(\mathbf{M}+1)$ ion and a neutral (M-1) fragment from the (2M) dimer.

When attempting to ionize the dimer out of the triplet state by delayed ionization with the $\mathrm{ArF}$ laser, after resonant excitation at the $0-0$ band of the dimer, only an ion signal at the monomer mass was observed. This is probably due to the fact that the triplet state dimer undergoes dissociation to a triplet and a ground state monomer, since its internal energy far exceeds that of the hydrogen bonds holding the dimer together.

\section{Clusters of PABA with molecules seeded in the beam}

Under the experimental conditions that turned out to be optimal for the production of jet-cooled PABA dimers, it was also possible to form and resonantly detect clusters of desorbed PABA molecules with molecules seeded in the jet expansion. In Fig. 11 part of the $(1+1)$-REMPI spectrum of the PABA-Ar van der Waals complex is shown. Again, this spectrum was recorded by monitoring the parent mass (177 amu). When a similar excitation and ionization efficiency for the PABA-Ar complex and the PABA monomer is assumed, we can conclude that we have roughly two orders of magnitude less PABA-Ar than PABA in the beam. The PABA-Ar shows a fairly large fragmentation ( $50 \%$ $80 \%$ ) into PABA monomers, even at the lowest ionization fluences used (in a single color experiment). The spectrum of the PABA-Ar vdW complex shows a red shift, as expected, of $42.5 \pm 0.5 \mathrm{~cm}^{-1}$ relative to the PABA spectrum. Two low-frequency van der Waals vibrations are clearly visible in the spectrum. Comparing the frequencies of these vibrations with those observed for the aniline-Ar complex, ${ }^{37}$ leads to the conclusion that the observed vibrations are the bending vibrations ( $b_{x}$ and $b_{y}$ ) of the $\mathrm{Ar}$ atom, sitting above the ring, 


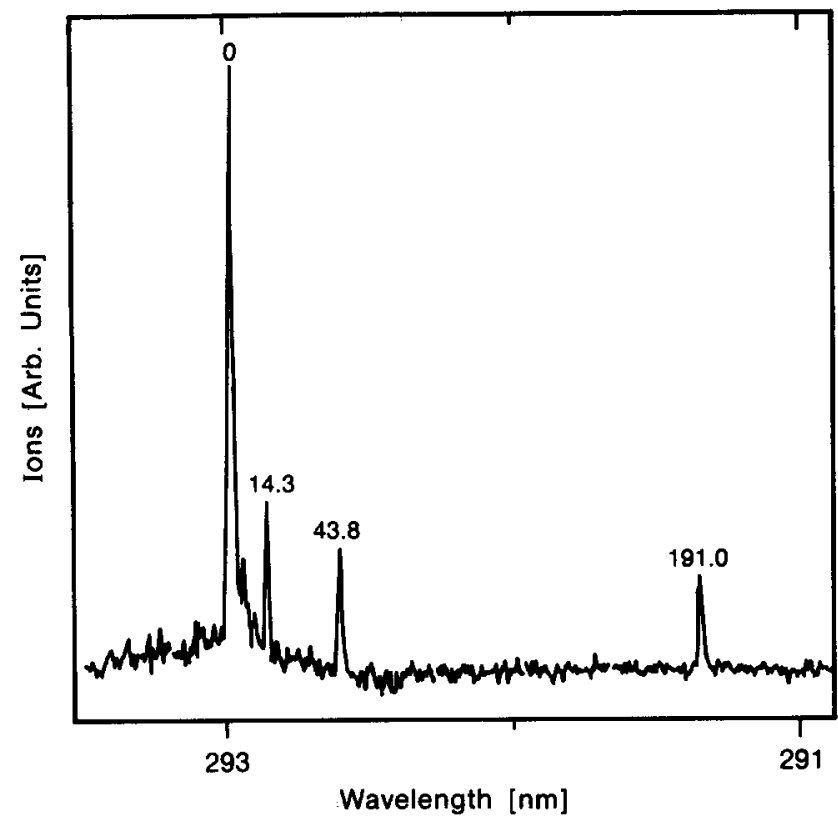

FIG. 11. $(1+1)$-REMPI spectrum of the PABA-Ar van der Waals complex, recorded at the parent mass ( $177 \mathrm{amu}$ ). The origin of the vdW complex is redshifted by $42.5 \pm 0.5 \mathrm{~cm}^{-1}$ from the PABA origin.

with respect to the ring. The lowest-lying strong vibration in the PABA spectrum ( $190.5 \mathrm{~cm}^{-1}$ from the origin) shows up with the same frequency in the spectrum of the PABA-Ar complex as well. The much stronger vibration at $461.2 \mathrm{~cm}^{-1}$ in the PABA spectrum, however, does not have its counterpart in the vdW complex. This puts the dissociation energy of PABA-Ar (in its electronic ground state) between 147 and $419 \mathrm{~cm}^{-1}$.

One of the problems in cluster spectroscopy is the fragmentation of clusters into smaller fragments. This causes spectra of higher clusters to show up at the mass of lower clusters, thus complicating the assignment of spectral features to specific cluster sizes. Although there are techniques to circumvent this problem, ${ }^{37,38}$ it is much less severe in the experimental setup we use. In the region where we produce the clusters, the pressure is orders of magnitude less than in a conventional cluster source. This means that the amount of higher order clusters present in the molecular beam decreases very fast with increasing cluster size.

We could record the spectrum of the (PABA-dimer)Ar complex on mass 314 . The signal intensity was just above the detection limit, and therefore the spectrum in Fig. 12 looks somewhat noisy. Only the region with the strongest signal, around the origin, is shown, but this is also the most interesting region. The (PABA-dimer)-Ar complex clearly shows a double origin; two peaks, separated by $37.8 \pm 1.0$ $\mathrm{cm}^{-1}$, having the same intensity, are visible. Compared with the origin in the PABA-dimer spectrum, the higher frequency peak in the (PABA-dimer)-Ar complex is red shifted by $5.4 \pm 1.0 \mathrm{~cm}^{-1}$. The lower frequency peak is shifted with respect to the PABA-dimer origin almost as much as the PABA-Ar origin is shifted with respect to the PABA origin, i.e., $43.2 \pm 1.3 \mathrm{~cm}^{-1}$ versus $42.5 \pm 0.5 \mathrm{~cm}^{-1}$.

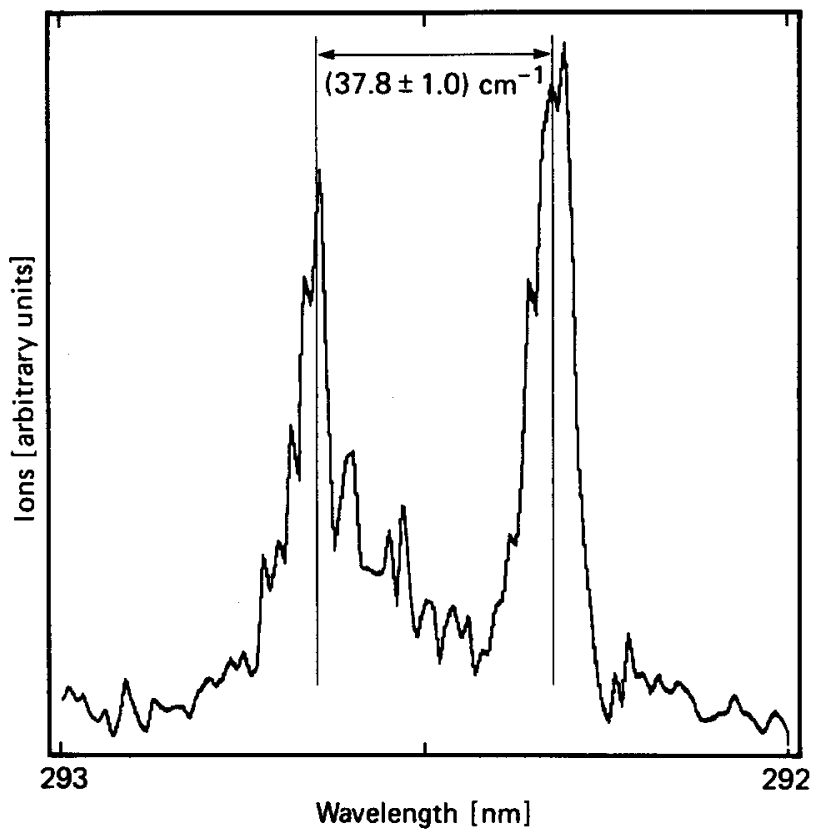

FIG. 12. Part of the $(1+1)$-REMPI spectrum of the (PABA-dimer)-Ar van der Waals complex, showing the double origin. Ions are recorded at mass $314 \mathrm{amu}$, the mass of the parent. The value that is indicated for the splitting of the bands, is averaged over five independent laser scans.

The appearance of the double origin for the (PABAdimer)-Ar complex can be explained by the previously demonstrated fact that the excitation in the PABA-dimer is strongly localized in one of the monomer units. The lower frequency peak is due to excitation of the monomer unit that has the Ar atom attached to it. Therefore this peak shows the same red shift as in PABA-Ar. The higher frequency peak shows hardly any red shift, and in this case the other monomer unit, the one which does not have an Ar atom above the ring, is excited. The reason why this peak is shifted from the PABA-dimer origin at all is a change in zero-point energy due to the Ar attached to the unexcited half of the complex.

Hydrogen-bonded complexes of PABA with methanol and water could be cooled and resonantly detected as well. The vapor pressures of methanol and water at room temperature are high enough to see a considerable amount of clusters. The signals for resonant detection of $\mathrm{PABA}-\mathrm{H}_{2} \mathrm{O}$ or PABA- $\mathrm{CH}_{3} \mathrm{OH}$ clusters were stronger than the signals at the PABA-Ar mass, even though the concentration of $\mathrm{H}_{2} \mathrm{O}$ and $\mathrm{CH}_{3} \mathrm{OH}$ in the beam was orders of magnitude less than the concentration of Ar. This might be partly due to the presence of an $\mathrm{H}$ bond instead of the weak vdW bond, and partly due to an increase in the lifetime of the $S_{1}$ state which increases the ionization efficiency. Such an increase in lifetime of the intermediate state upon complexation is observed in benzoic acid for example. ${ }^{31} \mathrm{~A}(1+1)$-REMPI spectrum of jet cooled PABA- $\mathrm{CH}_{3} \mathrm{OH}$ is shown in Fig. 13. Ions at the parent cluster mass (169 amu) were detected. There is a close similarity between this cluster spectrum and the PABA 


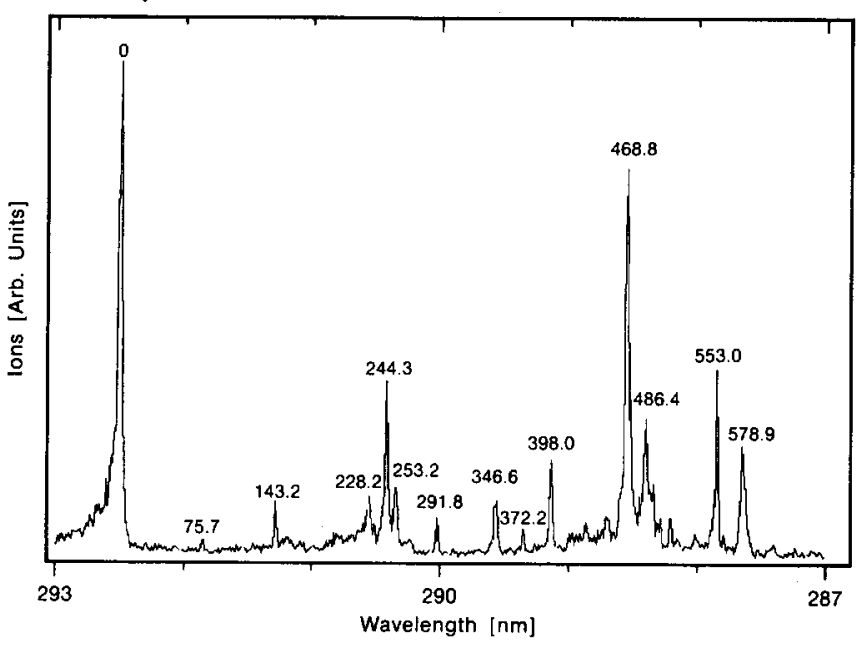

FIG. 13. $(1+1)$-REMPI spectrum of the PABA- $\mathrm{CH}_{3} \mathrm{OH}$ hydrogen bonded complex, recorded at mass 169 . Frequency distances of the various vibrations from the origin are indicated with $a \pm 1 \mathrm{~cm}^{-1}$ accuracy. The origin of this complex is $16.3 \pm 0.7 \mathrm{~cm}^{-1}$ blueshifted from the PABA origin.

spectrum shown in Fig. 3. The origin of the PABA- $-\mathrm{CH}_{3} \mathrm{OH}$ spectrum is $16.3 \pm 0.7 \mathrm{~cm}^{-1}$ blueshifted with respect to the PABA origin. At low frequencies (75.7 and $143.2 \mathrm{~cm}^{-1}$ ) the hydrogen bond vibrations are seen again. Apart from these hydrogen bond vibrations, the spectra of $\mathrm{PABA}-\mathrm{H}_{2} \mathrm{O}$ and $\mathrm{PABA}-\mathrm{CH}_{3} \mathrm{OH}$ are almost identical, and therefore only the latter is shown. All vibrations seen in the PABA spectrum are slightly blueshifted in the PABA- $\mathrm{CH}_{3} \mathrm{OH}$ complex. Clearly, some vibrations in the bare PABA are changed more upon complexation than others. The geometrical structure of the hydrogen-bonded complexes of PABA with methanol and water is somewhat similar to the dimer structure. Both hydrogen and methanol can form two hydrogen bonds with the carboxyl group in PABA. It is interesting that the puzzling pair of peaks that shows up in the dimer spectrum (Sec. III B) can already be recognized in the $\mathrm{PABA}-\mathrm{CH}_{3} \mathrm{OH}$ spectrum at 228.2 and at $253.2 \mathrm{~cm}^{-1}$. This shows explicitly that the appearance of the "doublet" in the dimer spectrum is not related to an exciton type of splitting, a conclusion that could also be reached from the spectra displayed in Fig. 9.

Although we used the laser desorbed molecule (PABA) as the chromophore in these clusters, it is also possible to use the molecules seeded in the beam as a chromophore. This opens up the possibility of attaching strong chromophores, seeded in the jet expansion, to laser-desorbed molecules that cannot easily be resonantly detected themselves. The cluster spectra, while still specific for the desorbed molecules, are all located near the spectrum of the chromophore, and the cluster mass indicates the mass of the desorbed molecules.

\section{CONCLUSIONS}

We have demonstrated that laser desorption jet cooling is a powerful technique that permits studying the gas-phase spectroscopy of large, nonvolatile, organic molecules. As a surface analytical method it is more powerful than ordinary laser desorption techniques, in that selective and efficient ionization can be performed, using resonant transitions in the internally cold molecules. Dimers of para amino benzoic acid, formed in the expansion region, were cooled and resonantly detected. The dimer spectra indicate clearly that the excitation in the dimer is strongly localized in the monomer units. The interaction energy $\epsilon$ between the monomer units in the dimer is small, $\epsilon=0.4 \pm 0.3 \mathrm{~cm}^{-1}$. The geometrical structure of the dimer in the gas phase is that of a symmetrical carboxylic acid dimer, similar to that of benzoic acid. Clusters of laser-desorbed molecules with molecules seeded in the molecular beam could be cooled and resonantly detected.

${ }^{1}$ M. A. Posthumus, P. G. Kistemaker, H. L. Z. Meuzelaar, and M. C. Ten Noever de Brauw, Anal. Chem. 50, 985 (1978).

${ }^{2}$ J. H. Hahn, R. Zenobi, and R. N. Zare, J. Am. Chem. Soc. 109, 2842 (1987).

${ }^{3}$ J. Grotemeyer, U. Boesl, K. Walter, and E. W. Schlag, J. Am. Chem. Soc. 108, 4233 (1986).

${ }^{4}$ Lasers in Mass Spectrometry, edited by D. Lubman (Oxford University, Oxford, 1990).

${ }^{5}$ R. J. Conzemius and J. M. Capellan, Int. J. Mass Spectrom. Ion Phys. 34, 197 (1980).

${ }^{6}$ R. B. Hall, J. Phys. Chem. 91, 1007 (1987).

${ }^{7}$ M. G. Sherman, J. R. Kingsley, J. C. Hemminger, and R. T. McIver, Jr., Anal. Chim. Acta 178, 79 (1985).

${ }^{8}$ J. H. Hahn, R. Zenobi, J. L. Bada, and R. N. Zare, Science 239, 1523 (1988).

${ }^{9} \mathrm{C}$. H. Becker, Conference on Ion Formation from Organic Solids V, Lovanger, Sweden, edited by A. Hedlin and B. U. R. Sundgvist (Wiley, New York, 1990), p.167.

${ }^{10}$ J. Grotemeyer, U. Boesl, K. Walter, and E. W. Schlag, Org. Mass Spectrosc. 21, 645 (1986).

${ }^{11} \mathrm{~J}$. Grotemeyer, U. Boesl, K. Walter, and E. W. Schlag, Org. Mass Spectrose. 21, 595 (1986).

${ }^{12}$ D. H. Levy, Science 214, 263 (1981).

${ }^{13}$ Liang Li and David M. Lubman, Rev. Sci. Instrum. 59, 557 (1988).

${ }^{14}$ J. R. Cable, M. J. Tubergen, and D. H. Levy, J. Am. Chem. Soc. 109, 6198 (1987).

15J. R. Cable, M. J. Tubergen, and D. H. Levy, J. Am. Chem. Soc. 110, 7349 (1988).

${ }^{16}$ P. Arrowsmith, M. S. de Vries, H. E. Hunziker, and H. R. Wendt, Appl. Phys. B 46, 165 (1988).

${ }^{17}$ G. Meijer, M. S. de Vries, H. R. Wendt, and H. E. Hunziker (to be published).

${ }^{18}$ G. Meijer, M. S. de Vries, H. E. Hunziker, and H. R. Wendt, in Digest of Topical Meeting on Laser Applications to Chemical Analysis, 1990 (Optical Society of America, Washington, D.C., 1990), Vol. 2, post-deadline contributions.

${ }^{19}$ J. C. D. Brand, D. R. Williams, and T. J. Cook, J. Mol. Spectrosc. 20, 359 (1960).

${ }^{20}$ N. Mikami, A. Hiraya, J. Fujiwara, and M. Ito, Chem. Phys. Lett. 74, 531 (1980).

${ }^{21}$ S.-I. Kamei, H. Abe, N. Mikami, and M. Ito, J. Phys. Chem. 89,3636 (1985).

${ }^{22}$ R. Sabbah, R. Chastel, and M. Lafitte, Can. J. Chem. 52, 2201 (1974).

${ }^{23}$ W. C. Wiley and I. H. McLaren, Rev. Sci. Instrum. 26, 1150 (1955).

${ }^{24}$ M. A. Duncan, T. G. Dietz, and R. E. Smalley, J. Chem. Phys. 75, 2118 (1981).

${ }^{25}$ R. Howell, A. C. Jones, A. G. Taylor, and D. Phillips, Chem. Phys. Lett. 163, 282 (1989)

${ }^{26}$ J. August, T. F. Palmer, J. P. Simons, C. Jouvet, and W. Rettig, Chem. Phys. Lett. 145, 273 (1988).

${ }^{27}$ G. Meijer, M. S. de Vries, H. R. Wendt, and H. E. Hunziker (to be published). 
${ }^{28}$ A. Oikawa, H. Abe, N. Mikami, and M. Ito, J. Phys. Chem. 88, 5180 (1984).

${ }^{29}$ T. R. Rizzo, Y. D. Park, and D. H. Levy, J. Chem. Phys. 85, 6945 (1986).

${ }^{30}$ D. E. Poeltl and J. K. McVey, J. Chem. Phys. 88, 4349 (1983).

${ }^{31}$ Y. Tomioka, H. Abe, N. Mikami, and M. Ito, J. Chem. Phys. 88, 2263 (1984).

${ }^{32}$ T. F. Lai and R. E. Marsh, Acta Crystallogr. 22, 885 (1967).
${ }^{33}$ J. C. Baum and D. S. McClure, J. Am. Chem. Soc. 101, 2335 (1979).

${ }_{34}^{34}$ J. C. Baum and D. S. McClure, J. Am. Chem. Soc. 101, 2340 (1979).

${ }^{35}$ J. C. Baum, J. Am. Chem. Soc. 102, 716 (1980).

${ }^{36}$ J. C. Baum and D. S. McClure, J. Am. Chem. Soc. 102, 720 (1980).

${ }^{37}$ E. J. Bieske, M. W. Rainbird, and A. E. W. Knight, J. Chem. Phys. 90, 2068 (1989).

${ }^{38}$ V. Beushausen, Ph.D. thesis, West Germany, 1989. 